\title{
Duplex scanning using sparse data sequences
}

\author{
Møllenbach, S. K.; Jensen, Jørgen Arendt
}

Published in:

IEEE Ultrasonics Symposium, 2008. IUS 2008.

Link to article, DOI:

10.1109/ULTSYM.2008.0002

Publication date:

2008

Document Version

Publisher's PDF, also known as Version of record

Link back to DTU Orbit

Citation (APA):

Møllenbach, S. K., \& Jensen, J. A. (2008). Duplex scanning using sparse data sequences. In IEEE Ultrasonics Symposium, 2008. IUS 2008. (pp. 5-8). IEEE. https://doi.org/10.1109/ULTSYM.2008.0002

\section{General rights}

Copyright and moral rights for the publications made accessible in the public portal are retained by the authors and/or other copyright owners and it is a condition of accessing publications that users recognise and abide by the legal requirements associated with these rights.

- Users may download and print one copy of any publication from the public portal for the purpose of private study or research.

- You may not further distribute the material or use it for any profit-making activity or commercial gain

- You may freely distribute the URL identifying the publication in the public portal

If you believe that this document breaches copyright please contact us providing details, and we will remove access to the work immediately and investigate your claim 


\title{
Duplex scanning using sparse data sequences
}

\author{
Sara Klingenberg Møllenbach and Jørgen Arendt Jensen \\ Center for Fast Ultrasound Imaging, Dept. of Electrical Engineering, DTU, Bldg. 349, \\ Technical University of Denmark, DK-2800 Kgs. Lyngby, Denmark
}

\begin{abstract}
The velocity distribution in vessels can be displayed using duplex scanning where B-mode acquisitions are interspaced with the velocity data. This gives an image for orientation, but lowers the maximum detectable velocity by a factor of two. Other pulse sequences either omits the B-mode image or leaves gaps in the velocity data, which makes it difficult to output audio data. The near full velocity range can be maintained and B-mode images shown by using a sparse data sequence with velocity and B-mode samples intermixed. The B-mode samples are placed in a (sparse) periodical pattern, which makes reconstruction of the missing samples possible. The periodic pattern has the length $T=M+A$ samples, where $M$ are for B-mode and $A$ for velocity estimation. The missing samples can now be reconstructed using a filter bank. One filter bank reconstructs one missing sample, so the number of filter banks corresponds to $M$. The number of sub filters in every filter bank is the same as $A$. Every sub filter contains fractional delay (FD) filter and an interpolation function. Many different sequences can be selected to adapt the B-mode frame rate needed. The drawback of the method is that the maximum velocity detectable is scaled by the factor $A / T$. The approach has been investigated using in vivo RF data from the Hepatic vein, Carotid artery and Aorta from a 33 year old healthy male. A B-K Medical 3535 ultrasound scanner has been used in Duplex mode with a BK 8556, 3.2 MHz linear array probe. The sampling frequency, the $f_{p r f}$ and the resolution are $15 \mathrm{MHz}, 3.5 \mathrm{kHz}$, and $12 \mathrm{bit}$ sample (8 $\mathrm{kHz}$ and 16 bit for the Carotid artery). The resulting data contains 8000 RF lines with 128 samples at a depth of $45 \mathrm{~mm}$ for the vein and $50 \mathrm{~mm}$ for Aorta. Sparse sequences are constructed from the full data sequences to have both a reference sequence and sparse data sequences. After reconstruction the reference and the reconstructed spectrum are almost identical when characterized by the Signal to Noise Ratio (SNR). This is investigated and optimized by altering the number of filter coefficients, the implementation of the fractional delay filter, and the sparse sequence. The Hepatic vein data are processed with 5 filter coefficients, a FD filter implemented with a Knab window and sequence length $T$ of $10 \mathrm{RF}$ lines. By removing 7 lines the SNR is calculated to be $30 \mathrm{~dB}$. When reconstruction over half the RF lines possible then to two spectograms can be acquired at the same time. The investigation of Aorta shows, that because the spectrum is wider, it puts some restrains on the selection of the sequence. The shortest sequence for getting a good spectrum consists of 7 lines, with one missing line $(14.3 \%, S N R=31.6 \mathrm{~dB})$. Using sparse sequences both $B$-mode and velocity data can be acquired with only a modest degradation in maximum velocity. The reconstruction gives errors below the normal noise level in velocity data, and the full audio signal is precisely reconstructed from the data.
\end{abstract}

\section{INTRODUCTION}

Ultrasound is a non invasive medical imaging modality, which in duplex scanning can make an anatomic B-mode image and simultaneously calculate and display the velocity distribution over time. Generating the B-mode image will im- pact the time for velocity estimation and lower the maximum detectable velocity. The purpose of the paper is to present a method for both preserving a high velocity range and at the same time be able to display a high frame rate B-mode image.

Recording a number of RF-lines after each other will generate a signal sampled with the pulse repetition frequency $\left(f_{p r f}\right)$ in which the frequency is proportional to the blood velocity [1]:

$$
f_{p}=f_{0} \frac{2|v| \cos (\theta)}{c}
$$

$f_{p}$ is the signal's frequency, $v$ is the blood velocity, $c$ is the speed of sound in the tissue. $f_{0}$ is the center frequency of the transducer and $\theta$ is the angle between the beam and the vessel. This can be used to display the velocity distribution by performing a Fourier transform of the received signal to show this in a gray shaded spectrogram.

Without the B-mode image the spectrogram is not useful, since it would not be possible to identify where the velocities were found. Both types of images are obtained simultaneously and in real time, which creates problems. In duplex scanning one emission is used in the B-mode image and another emission will give a RF-line for the velocity estimation. The transducer can only receive and transmit in one direction at a time, so it is necessary before scanning to make a strategy for how to distribute the pulses.

According to the sampling theorem the highest detectable frequency of the sampled signal is determined by the sampling frequency $\left(f_{p r f}\right)$ :

$$
f_{\max }=\frac{f_{p r f}}{2}
$$

yielding a maximum velocity of

$$
v_{\max }=\frac{c}{2 \cos (\theta)} \frac{f_{p r f}}{2 f_{0}}
$$

If the distribution strategy of the pulses for B-mode images and velocity estimations are evenly split, the maximum detectable velocity will be halved as a result of the decreased sampling frequency. Other distribution strategies leave holes in the spectrogram and prohibit an audio representation. Looking at the sampled signal for velocity estimation, the pulses that are used in the B-mode image leaves zero amplitude sampling points in the sampled signal. With the right distribution of pulses, these zero amplitude sampling points will be distributed in a periodical pattern. This signal can be described as periodically non-uniformed sampled. Bariska has in [2] described how the missing samples in such a signal can be reconstructed. Applying this method in medical ultrasound gives a solution to the problem in duplex scanning. 


\section{METHOD}

The reconstruction of a periodically non-uniformed sampled signal is described in [2], [3] and [4]. But [2] gives a method that is easy to implement and that only reconstruct the missing samples and not the continuous signal. The periodically nonuniform sampled signal can be divided into a number of sequences with the length $T$, where the pattern is non-uniform. The number of missing samples is $M$ and the number of available samples is $A$. The sum of $A$ and $M$ is $T$. This method of reconstruction is based on the use of filter banks. This filter bank interpolates the available signal values to get the missing signal values.

A filter bank is needed to make the reconstruction of the missing samples by interpolating between the available samples to generate the missing values. An example of a filter bank can be seen in Fig. 1. Here the full sampled signal is

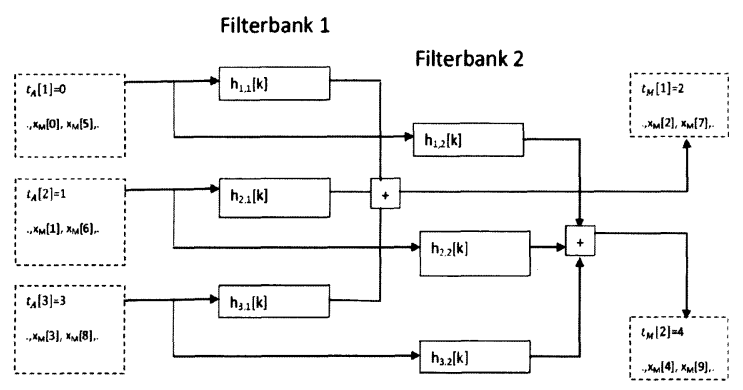

Fig. 1. From [2], an eksampel of a reconstruction filter bank

denoted $x$, the signal with periodically missing samples is denoted $x_{M}$ and the reconstructed signal is denoted $x_{R}$. A vector $t_{A}$ gives the time instances for the available samples in one sequence of $x_{M}$. A vector $t_{M}$ gives the time instances for the missing samples of one sequence in $x_{M}$. By using these vectors the relationship between $x_{M}$ and $x$ is given as:

$$
x\left(t_{A}+p T\right)=x_{M} \quad p=1,2, \cdots N
$$

This filter bank reconstructs the missing samples in the signal $x_{M}$ with a sequences length of 5 . The periodical pattern of the sequence is two available samples one missing, one available and one missing sample. It is here denoted a $[\mathrm{v} \mathrm{v} \mathrm{b}$ $\mathrm{v} b$ ] sequence, where $\mathrm{v}$ denotes a velocity emission and $\mathrm{b}$ a B-mode or other emission.

There is one filter bank for every missing sample, and one sub-filter for every available sample. Every filter in the filter bank can be described by a prototype filter that varies with every sub-filter. The filter bank is a synthesizing filter bank, with input signals of uniformly sampled sub-signals of available samples. These sub-signals are filtered and summed to find the signal of uniformly sampled missing samples. The result of the filtration is $M$ number of uniformly sampled signal consisting of the reconstructed values. Using these, the full reconstructed signal can be obtained.

Every sub-filter in the filter bank has a impulse response given as:

$$
h_{a}[k]=\operatorname{sinc}\left(\frac{t_{m_{1}}-t_{a}}{T}+k\right)(-1)^{k(A-1)} \prod_{q=1 q \neq a}^{A} \frac{\sin \left(\frac{\pi}{T}\left(t_{m_{1}}-t_{q}\right)\right)}{\sin \left(\frac{\pi}{T}\left(t_{a}-t_{q}\right)\right)}
$$

where $h$ is the impulse response of the filter, $A$ indicates the number for the available sample in the sequence, $k$ is the coefficient number, $t$ is the time instants, $m 1$ indicates the number for the missing sample in the sequence and $q$ is a counter.

To get a better understanding of the impulse response, it can be divided into three subsections. The sinc function is a low-pass filter. The "-1" makes a spectral inversion depending of $k$ (the number of filter coefficients) and $A$, according to [2]. The term that includes the product sign is the fractional delay. A fractional delay (FD) filter is a FIR filter that reconstructs samples within the sample instances. The low-pass FD filter can be described as:

$$
D_{\Delta}[k]=\operatorname{sinc}(\Delta+k),
$$

where $\Delta$ is the delay and $k$ is the vector $[-K ; K]$, and the number of filter coefficients is the length of the vector. $\Delta$ is determined by:

$$
\Delta=\frac{t_{m 1}-t_{a}}{T}
$$

$\Delta$ is not an integer and before implementing the sinc function it must be approximated. An easy way of implementing an approximation of the sinc function is by windowing the infinite impulse response, which is here done by a Knab window.

The price paid for interpolating samples is that the bandwidth of the reconstructed signal is reduced to:

$$
B \leq \frac{A}{T} \frac{f_{p r f}}{2} .
$$

Having e.g. $A=3$ available samples of a total of $T=5$ samples, thus, gives a velocity range reduced to $3 / 5$ of the range of a fully sampled sequence. The methods can, thus, be used to make any ratio between the time spend of B-mode and flow imaging at a corresponding reduction in maximum velocity.

\section{RESULTS}

The results are evaluated in two ways. One is the visual comparison of spectrograms made from a full sampled data sets and a reconstructed data set $\left(x_{R}\right)$. The signal with periodically missing samples is obtained by removing values from the fully sampled signal. The other way of evaluating the resulting reconstruction is by the Signal-to-Noise Ratio (SNR). This is the ratio between original signal and the difference between the reconstructed signal and the original signal:

$$
S N R=\frac{10 \log \left(P_{\text {Original }}\right)}{10 \log \left(P_{\text {recon }}-P_{\text {Original }}\right)}=\frac{10 \log \left(P_{\text {Original }}\right)}{10 \log \left(P_{\text {Noise }}\right)}
$$

All processing is done in MATLAB. When applying the reconstruction method the sequence are as described above, with two available samples, one missing, one available and one missing sample. The same filter bank is used as in Fig. 1. The number of filter coefficients is 7 , and the window used 
to implement the low pass filter is a Knab window [5] as recommended by [2].

The in-vivo acquired data used to investigate the reconstruction, are obtained from three different vessels: the Hepatic vein, the Carotid artery, and the Aorta. The data from the different vessels are obtained by measurements done by a ultrasound scanner B-K Medical type 3535 (B-K Medical Aps, Herlev, Denmark) and a B-K 8556,3.2 MHz linear array probe transducer. where the Scan mode was duplex. The B-mode frame rate was $27 \mathrm{f} / \mathrm{s}$ and the pulse repetition frequency was $3.5 \mathrm{kHz}$. for the Hepatic vein and the Aorta. The RF sampling frequency and resolution was $15 \mathrm{MHz}$ and 12 bit samples. For the Carotid artery only the analog signal out the scanner was sampled at $8 \mathrm{kHz}$ and 16 bit samples. The immediate visual results from the reconstruction of the data obtained by the Hepatic vein and the Carotid artery are seen in Fig. 2. No visible difference between the original signals spectrogram and the reconstructed signals spectrogram is seen.
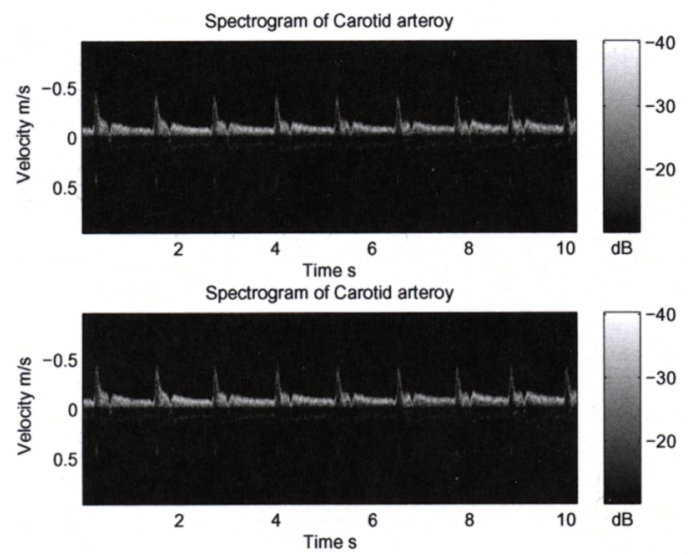

Fig. 2. The spectrogram of the original (on top) and reconstructed(bottom) Carotid signal

When the reconstruction method is used on Aorta data, there is some visible aliasing. This can also be seen by the SNR values. For the reconstruction of Hepatic vein data the SNR is $33.5 \mathrm{~dB}$. For the Carotid artery it is $45.2 \mathrm{~dB}$. The SNR from the failed reconstruction of the Aorta is $26.4 \mathrm{~dB}$ indicated aliasing for the reconstructed data.

The method can be optimized. The first parameter is the window used to restrict and implement the low pass filter. 17 different filters are tested with all three data sets. The window that led to the highest SNR in all three cases is the Knab window. The next parameter to be optimized is the number of filter coefficients. The SNR is found with different filter coefficients between 3 and 51 (only odd numbers). The resulting SNR shows that the number of coefficients does not affect the SNR much. There is a global max for all three data sets. The maximum is all found with a low number of coefficients, respectively 5, 19 and 3 for Hepatic vein, Carotid artery, end Aorta. Each of the global maxima was applied in the following study.

The next investigations are on variations of the sequences.
Three things about the sequences can be altered:

- The length of the sequence T

- The distributing of missing and available samples

- The permutation of the placement

An investigation of the relationship between the missing and available samples can be expressed by the percentage of missing samples in the sequence. In this investigation the missing samples are placed first in the sequence followed by the available samples. This investigation is done to sequences of different lengths. The result is shown in Fig. 3.

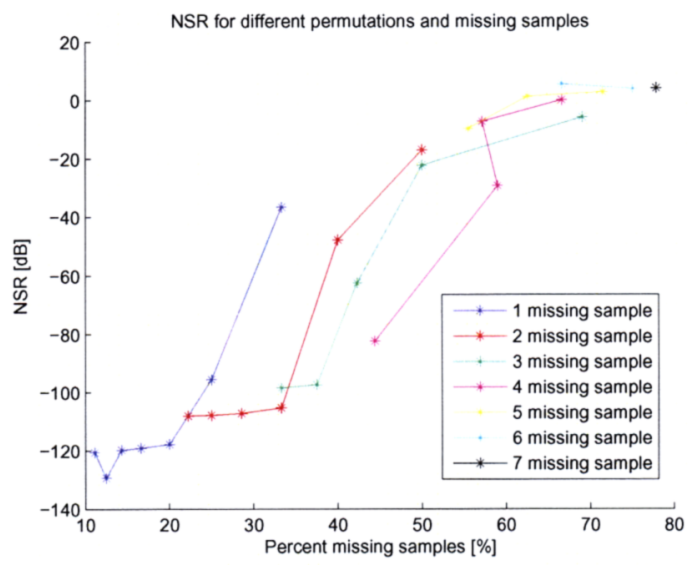

Fig. 3. Displayes the NSR as a function of the percentage of missing samples

It proved useful to work with NSR (Noise-to-Signal Ratio) rather than SNR.The NSR is the reciprocal of SNR. Fig. 3 shows the relationship between the percentage of missing samples and the NSR. Two lines can be drawn, the lowest line is where the noise is so small that it is under the machine precision and the top line is where there is nothing left of the signal but noise. By plotting the spectrogram of these signals it is seen that the aliasing will appear when $50 \%$ of the signal is missing. To explore this further the sequence length is doubled and the permutation is changed. By making the length of the sequence double, a non aliased spectrogram is made. This is also the case if only the permutation is changed. On this basis the permutation is investigated. A sequence length of 10 samples is used, the permutation is then altered. First 9 available samples with 10 combinations of sequences are used. The SNR is calculated and compared. This is done by removing another sample, finding the combinations until only 2 samples are available. The study showed that the permutation pattern have a big influence on the reconstruction. It is the same type of permutation pattern that led to the best results for all three data sets. It is also the same type of permutations that led to the worse reconstructions. It turns out that the permutations have more influence on the reconstruction than the number of available samples. Looking at the best permutations it is seen that the sequence with the best result is where the available and missing samples were intermixed. As an example the best sequence for the Hepatic vein and Carotid artery with 6 out of 10 missing 
samples was [v,b,v,b,b,v,b,v,b,b] and [b,b,v,b,v,b,b,v,b,v]. The permutations that gave the lowest SNR are the permutations where the available and missing samples are clustered together. As an example the worst sequence for Hepatic vein and Carotid artery with 4 out of 10 missing samples was [v,b,b,b,b,b,b,v,v,v] and [v,v,b,b,b,b,b,b,v,v]. To sum up the investigation, the spectrograms with a sequence length of 10 and the lowest number of available samples that give a satisfactory result is found. For the data from the Hepatic vein the only data needed is 3 available samples $(30 \%)$ and the responding spectrogram are seen in Fig. 5. The SNR of the signal is $29.8 \mathrm{~dB}$.

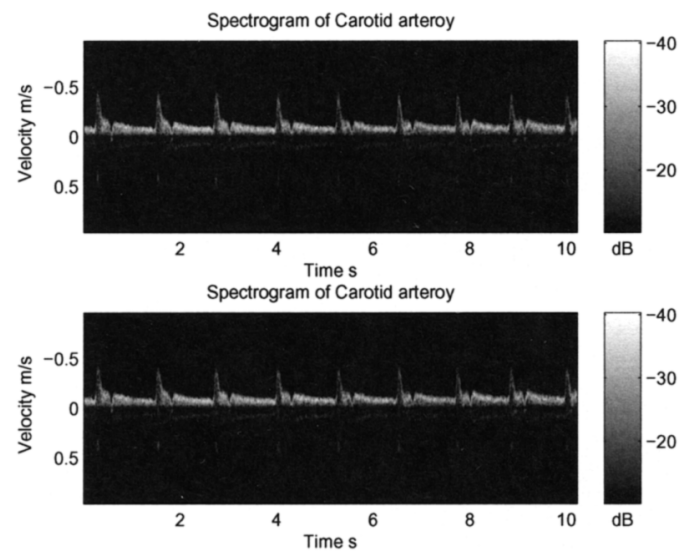

Fig. 4. The spectrogram of the fully sampled and reconstructed Carotid signal. Used sequence $=[\mathrm{b}, \mathrm{b}, \mathrm{v}, \mathrm{b}, \mathrm{v}, \mathrm{b}, \mathrm{b}, \mathrm{v}, \mathrm{b}, \mathrm{v}]$

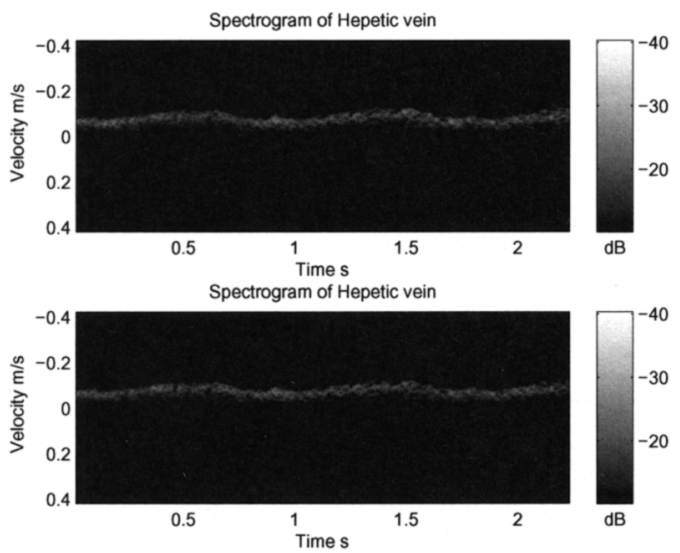

Fig. 5. The spectrogram of the fully sampled and reconstructed Hepatic signal. Used sequence $=[\mathrm{v}, \mathrm{b}, \mathrm{b}, \mathrm{v}, \mathrm{b}, \mathrm{b}, \mathrm{v}, \mathrm{b}, \mathrm{b}, \mathrm{b}]$

The same is done for the data from the Carotid artery. The reconstruction is successful when $40 \%$ of the data are available, and give a SNR of $23.4 \mathrm{~dB}$. By using the data from the Aorta it is only possible to make a satisfactory spectrogram if $90 \%$ of the data are available. To improve this, the sequence length is made smaller (the percentage of available samples

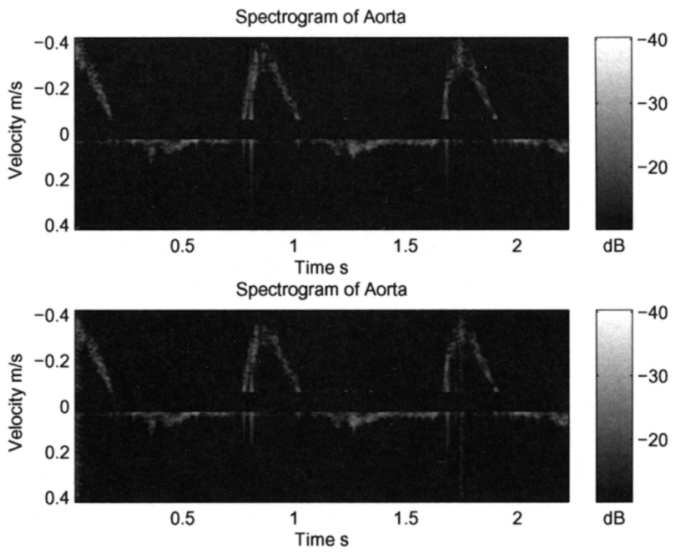

Fig. 6. The spectrogram of the fully sampled and reconstructed Aorta signal. Used sequence $=[\mathrm{v}, \mathrm{v}, \mathrm{v}, \mathrm{b}, \mathrm{v}, \mathrm{v}, \mathrm{v}]$

made lager). The lowest percentage of available data to make a good spectrogram is $86 \%$. This signal has a SNR of 31.6 dB.

\section{CONCLUSIONS}

The method can be used to reconstruct missing samples in signal used to make the spectrogram. It is hereby possible to select any ratio between B-mode lines and spectrogam lines to tailor the frame rate of the image and the velocity range of the spectrogram. The penalty is the proportional reduction in maximum velocity that can be displayed, This offers a possible more sound approach than gap filling, where the Bmode image is acquired, The performance of the method is highly dependent on the sparse data sequence used and the interpolation filters and it might be possible to further optimize these. Also studies in an in-vivo setting is needed to reveal the potential drawbacks of the sparse emissions, which might give rise to reverberations. However, good results are obtained when the data is within the aliasing range, and it is not possible visually toe distinguish between a sparse and full spectrogram.

\section{REFERENCES}

[1] J. A. Jensen. Estimation of Blood Velocities Using Ultrasound A Signal Processing Approach. Cambridge University Press, 1996.

[2] A. Bariska. Recovering periodically spaced missing samples. IEEE Signal Processing Magazine, pages 127-129, 2007.

[3] R. W. Adams. Nonuniform sampling of audio signals. J. Audio Eng. Soc, 40:886-893, 1992.

[4] Y. C. Eldar og A. V. Oppenheim. Filterbank reconstruction of bandlimited signals from nonuniform and generalized samples. IEEE Trans. on Signal Processing, 48:2864-2875, 2000.

[5] Knab. J. An alternate keiser window. IEEE Trans. on Acoustics, Spech, and Signal Processing, ASSP-27, No. 5:560-561, 1979. 